\title{
NOTAS A LAS LECCIONES DE HUSSERL SOBRE LA CONCIENCIA DEL TIEMPO ${ }^{1}$
}

Es bien sabido que el concepto de intencionalidad, que es el concepto central de la fenomenología, recibe una nueva clarificación y extensión en las lecciones de Husserl sobre la fenomenología de la conciencia interna del tiempo. Según aceptación propia de Husserl, el análisis de la intencionalidad, como puede encontrarse en las Ideen I, es realizado dentro de una limitación impuesta por si mismo. Ya en sus lecciones sobre la conciencia del tiempo, que preceden en fecha a las Ideen, Husserl había ido más allá de estos límites, pero en ellas parece haber decidido no hacer uso de esas exploraciones. Así, aunque las lecciones preceden en fecha a las Ideen, con todo en madurez filosófica marcan ellas un desarrollo ulterior. En este artículo vamos a examinar críticamente algunas doctrinas clave de las lecciones. Sin embargo, no nos vamos a referir al tema central de la conciencia del tiempo, excepto en tanto que conduce al concepto de intencionalidad. Nuestras notas se limitarán a la situación de las lecciones sobre este concepto clave, y así no han de tomarse como una evaluación general de las lecciones tomadas como un todo.

Martín Hidegger, quien editó estas lecciones, escribe en el prólogo que su tema central es la constitución temporal del dato puro de la sensación (Empfindungsdatum) y la autoconstitución del "tiempo fenomenológico", que se halla a la base de una constitución semejante. Heidegger añade que esto no solamente descubriría el carácter intencional de la conciencia del tiempo, sino que contribuirfa en orden a una clarificación básica de la intencionalidad en general. El concepto de intencionalidad necesita una clarificación semejante, pues la palabra 'intencionalidad', aún ahora, de acuerdo con Heidegger, "no es una palabra que resuelva todos los problemas, sino el título de un problema capital". 2 Desde este punto de vista es desde donde habremos nosotros de enfocar las lecciones en este artículo.

Trataremos primeramente de plantear el problema de Husserl. En las Ideen I se nos dice que el noema es constituido por los actos noéticos, que confieren significado, sobre la materia hilética. No se había dado ningún aná-

1 Edmund Husserl, Vorlesungen zur Phänomenologie des inneren Zeitbewusstseins, herausgegeben von Martin Heidegger, Halle, 1928. (En adelante será citada como ZBW).

$2 \mathrm{ZBW}, 367$. 
lisis de los datos hiléticos. El análisis que se había dado de los actos noéticos es algo estructural en la naturaleza; gracias a las Logische Untersuchungen tenemos el análisis de actos en su materia intencional, cualidad de acto y la esencia intencional resultante. En las Ideen I el noema fue analizado en sus bases estructurales correspondientes. Pero el sentido en que se había dicho que el noema era constituido por los actos y los datos hiléticos, es muy diferente del sentido en que un acto es descifrado desde su materia intencional, cualidad intencional y esencia intencional. De hecho, el problema de la constitución de actos, como también de los datos hiléticos, se dejó fuera de la discusión. Se tiene la impresión de que el problema de la constitución no puede del todo plantearse significativamente con respecto a ellos, pues los actos son las fuentes originales de significado, los que confieren significado $y$ así no puede decirse que ellos mismos hayan derivado su unidad de alguna otra fuente, mientras que los datos hiléticos son precisamente material no significativo y así no poseen ninguna significación por sí mismos, que pudiera tal vez remontarse hasta alguna otra fuente constitutiva. Pues o son en su facticidad y en su irracionalidad elementos de nuestra experiencia absolutamente primitivos, no analizables ulteriormente o requieren alguna especie de explicación causal y naturalista, que cae fuera dèl alcance de una filosofía fenomenológica, habiendo sido desechada por la epoché. Entonces los datos hiléticos parecerían ser elementos absolutamente primitivos, no significantes en sí mismos y privados en esa forma de toda unidad u objetividad. Los actos son los que confieren significado, unidad y objetividad a ellos. Los actos son primitivos en un sentido muy diverso. Éstos no son constituidos, porque constituyen. Si a toda esta doctrina de Husserl en las Logische Untersuchungen añadimos que sólo los actos objetivantes (objektivierende) son los portadores primarios de significado, de manera que los actos no-objetivantes presuponen a los objetivantes en orden a engendrar alguna clase de objetividad propia, obtenemos que la visión resultante es que toda objetividad es constituida por los actos objetivantes, es decir, por actos de aprehensión (Auffassung), que trabajan sobre el material bruto provisto por los llamados datos hiléticos. Este nítido esquema es el que se derrumba en las lecciones sobre la conciencia del tiempo. Ahora Husserl plantea el problema acerca de la constitución tanto de los actos como de los datos hiléticos considerados como objetos inmanentes y en el proceso de contestar a esas preguntas describe niveles de intencionalidad más profundos de lo que puede ser llamado intencionalidad-acto. 
En esta forma nos encaramos con tres diferentes niveles del problema de la constitución y por ello también del concepto de intencionalidad. ${ }^{3}$

1. La constitución del noema trascendente;

2. La constitución de las unidades inmanentes;

3. La autoconstitución del flujo absoluto de la conciencia.

El poema trascendente, como se ha mostrado en las Ideen I, es constituido por los actos y los datos hiléticos, pero ahí el problema de su constitu. ción había sido tratado en una forma que deja el problema de la duración completamente fuera del cuadro. De hecho, sin embargo, los objetos trascendentes son objetos durables, y el problema de su constitución es sólo el otro lado de la cuestión de cómo objetos durables se convierten en objetos de la conciencia. La última cuestión se divide en las siguientes: ccómo se tiende en la conciencia hacia objetos durables, y cómo tales intenciones llegan a realizarse? Ahora bien, Husserl reconoce, como Kant, que ser identificable en el recuerdo es una parte del significado de la objetividad temporal. ${ }^{4}$ De hecho, el objeto es lo que puede ser identificado en muchos actos diferentes. Pero semejante posibilidad de identificación es la función, y por ello no es extraño que Husserl considere la identidad de un objeto temporal como si fuera constituida mediante ciertas posibles coincidencias de identificaciones por medio de recuerdos. Esto a su vez se conecta con el hecho-derivable de la naturaleza de la conciencia del tiempo inmanente- de que aun cuando cada "ahora" se hunde en el pasado junto con sus retenciones y protensiones, es decir, con su total horizonte intencional, su posición temporal permanece inalterada. Lo que cambia es su modo de ser dado y también su intervalo temporal desde el ahora actual.5 Este hecho de que cada "ahora", a pesar de su hundimiento hacia atrás en el pasado, retiene inalterada su precisa posición temporal, y el otro hecho de que nosotros podemos, en la conciencia reproductiva, identificarlo en actos repetidos, son solamente dos lados -objetivo y subjetivo- del mismo fenómeno. Esto solo, sin embargo, no daría la razón de la posibilidad de tender hacia o aprehender objetos temporales durables. Ya hemos alcanzado la posibilidad de identificar la posición temporal, pero necesitamos también dar razón de la posibilidad de identificar el contenido extratemporal del objeto. Cuando ambos son preservados sin alteración, como se hace patente por la conciencia reproductiva, tenemos

3 Cfr. "La doctrina de la constitución es un intento de pensar sobre el enigma de la intencionalidad." (R. Sokolowski, The Formation of Husserl's Concepts of Constitution, The Hague, 1964, p. 135.)

4 "Identität von Zeitobjekten ist also ein konstitutives Einheitsprodukt gewisser möglicher Identifizierungsdeckungen von Wiedererinnerungen." (ZBW, 460.)

6 $\mathrm{ZBW}, 4^{18-420,} 4^{60}$. 
un objeto durable. En otras palabras, podemos decir que los objetos durables y trascendentes son constituidos por los actos reproductivos de identificación sobre la base de contenidos extra-temporales. Algunos recuerdos son del mismo objeto, cuando concuerdan con respecto a su contenido intencional, es decir, con respecto a sus contenidos extra-temporales más el horizonte temporal. Decir que algo es una cosa individual durable, vale decir que es capaz de ser el contenido intencional común de un número indefinido de tales actos recordatorios identificantes. "El objeto es una unidad de conciencia que en actos repetidos puede aparecer como el mismo." 6

Aun cuando la teoría de la constitución de los objetos trascendentes ha dado así un rasgo característico temporal, lo que es realmente nuevo en estas lecciones es el problema de la constitución de las unidades inmanentes. Los actos y los datos hiléticos son también objetos en un cierto sentido. Pues seguramente son objetos posibles de reflexión, sea de la percepción interna o de la abstracción analítica o de la mirada reflexiva. Y en tanto son objetos, necesitan ellos mismos un análisis intencional de su constitución. Además, son unidades temporales y pertenecen a lo que Husserl llama tiempo inmanente, pre-objetivo. En la búsqueda de su constitución, estamos verdaderamente destinados a sumergirnos en niveles más profundos de intencionalidad.

$\mathrm{Ni}$ los actos ni los datos hiléticos son apariencias de ninguna otra cosa. Sin embargo, se muestra que están extendidos temporalmente, y aquí reside la posibilidad de exponer su constitución. Objetos inmanentes son formados de fases temporales, y cada una de estas fases temporales se caracteriza tanto por una referencia retentiva a lo que ha sido, como por una referencia protentiva a lo que está aún por ser, y el objeto inmanente total, acto o dato hilético, es lo que es, sólo en tanto que, durando su duración actual, posee continuamente estas referencias hacia atrás y hacia adelante. ${ }^{7}$ Husserl parece estar delineando más bien una dudosa distinción entre "aprehensiones originarias" (Urauffassungen) o "impresiones originarias" (Urimpressionen), las cuales constituyen el objeto plenamente inmanente y el acto total o dato que es constituido por este medio. El acto plenamente intencional, en el sentido de las Ideen I, está constituido por decirlo así de intenciones parciales, y estas mismas intenciones parciales no están constituidas, sino que pertenecen al flujo básico de la conciencia. ${ }^{8}$

¿Qué quiere decir Husserl con esta última distinción? Podría haber que.

B "Das Objekt ist eine Einheit des Bewusstseins, die in wiederholten Akten (also in zeitlicher Folge) sich als dieselbe herausstellen kann..." (ZBW, 461.) P. F. Strawson en su Individuals, An Essay in Descriptive Metaphysics (Methuen, London, 1959) percibe esta relación entre la naturaleza de los objetos individuales y el problema de la identificación. Pero la orientación de Strawson en el problema de la identificación es espacial y lingüistica, mientras que la de Husserl es temporal y fenomenológica.

7 ZBW, $433-436$.

8 Sokolowski, loc. cit., 86. También ZBW, 444. 
rido decir que un acto total, como la percepción de una casa, consiste en muchos actos parciales de percibir diferentes perfiles en ella, y éstos a su vez de muchas sensaciones primarias. El acto total en este caso es constituido por esos actos parciales, pero al fin y al cabo por esas sensaciones primarias. Mas esto sería una especie de análisis estructural de un acto intencional, que parece incompatible con el espíritu del análisis fenomenológico. Ello implicaría el análisis de un acto total en lo que se supone ser sus elementos constitutivos. Además, es altamente dudoso, desde un punto de vista fenomenológico, decir que mi acto de percibir una casa consiste en muchos actos par. ciales diferentes de percibir los varios perfiles de la casa. Los perfiles no son lo que yo percibo, yo percibo a través de ellos. Los actos dirigidos a los perfiles no constituyen el acto de percibir el objeto total, si bien puede decirse que hacen posible lo último. Puede decirse que el acto total es construido sobre los actos parciales. Se puede aún ir más lejos, y decir que la intención total está presente en cada una de las intenciones parciales y las hace lo que son, y no viceversa.

Más en consonancia con lo que Husserl hace en las lecciones sobre la conciencia del tiempo es la interpretación siguiente: cada acto es un objeto temporal en el sentido de que tiene una duración en el tiempo. El acto de percepción, por ejemplo, tiene una extensión en el tiempo, por más pequeña que sea. Ahora bien, si tiene una extensión en el tiempo, es analizable en series de "ahoras", teniendo cada ahora su propio horizonte retencional y protensional, y hundiéndose hacia atrás en el pasado junto con ese horizonte. Estos ahoras son los que constituyen las impresiones originarias, y no son constituidos ya más, si bien constituyen, al fin y al cabo, a todos los otros objetos, inmanentes o trascendentes.

Se ha argüido rectamente por Sokolowski que este análisis formal de la constitución de un acto deja sin explicar por qué un acto tendría esta-cualidad más bien que ésa. En otras palabras, ello no nos provee de una constitución de la cualidad específica del acto. A saber, de su cualidad como acto de juicio o como acto de deseo o anhelo, aunque en el mejor caso tenemos aquí una estimación de la posibilidad del acto como unidad temporalmente extendida. ${ }^{9}$ Yo forzaria todavía más el punto, y sostendría que esto no nos provee aún de una adecuada constitución del acto como acto intencional. En primer lugar, no es del todo claro en qué sentido cada acto intencional, como objeto inmanente, tiene una extensión temporal. El acto de escuchar una melodía continua puede ser un acto que tenga dilatación temporal. Pero el acto de percibir una casa seguramente no tiene historia temporal. A su vez, puede decirse en cierto sentido que el recordar se extiende temporalmente, en particular en cuanto que excitan dentro de él mismo intenciones que deben ser realizadas, y puede de hecho consistir en la realización progresiva de tales

0 Sokolowski, loc, cit., 92-93, 100. 
intenciones. La introspección no es siempre una guía segura en estas materias. Pues los actos pueden tener dilatación temporal o pueden no tenerla. $Y$ pueden estar, como parecen estarlo los actos de percepción, concentrados en un ahora temporal. Seguramente esto no es negar que los actos de percepción despiertan además intenciones que deben ser realizadas progresivamente en el tiempo. Esto no es tampoco negar que el ahora en que un acto de percepción está, por decirlo así, concentrado, posee su horizonte retencional y protensional. Lo que yo niego es que todos los actos puedan ser resueltos en fases temporales. Un acto de percepción no puede ser resuelto así. El acto total, como acto total, está en el ahora. El acto, considerado como acto intencional, no es un proceso, no tiene duración. La intención es puntiforme. Las intenciones componentes o las intenciones resultantes no rompen la puntiformidad de la intención total. Las intenciones parciales, sobre las que se construye una intención total, no son fases de ella, son su soporte y sus medios. Una intención puede dar origen o despertar intenciones ulteriores, puede presionar hacia adelante a su realización, y en tal medida la temporalidad entra en su constitución. Pero no podemos precisamente analizarla en fases e intentar derivar su constitución del mutuo sumergirse de estas fases en otra en virtud de sus horizontes retencionales y protensionales.

De hecho, me parecería a mí que cuando Husserl habla de la "constitución" del noema trascendente en los actos y también de la "constitución" de los actos inmanentes èn fases temporales, no está usando la palabra "constitu. ción" precisamente en el mismo sentido. En el primer caso, el sentido de "constitución" es genuinamente fenomenológico, pues no significa un análisis de un noema en sus fases constituyentes o partes, sino que lo implica, haciendo remontar el noema a los actos a los que tiende. En el segundo caso, estaríamos simplemente descomponiendo un acto total en aquellas fases que van a formarlo: el análisis, por tanto, parece ser real más bien que intencional.

Me parecería a mí, por tanto, que el acto es constituido no por fases temporales, sino por aquellas otras intenciones más simples, si es que existen algunas semejantes, las cuales en todo caso permanecerían anónimas detrás de él. Todo lo que Husserl podría decir legítimamente es que son esas intenciones más simples, más primitivas, las que constituyen convenientemente el tiempo, o las que son las intenciones básicas, fuera de las cuales se constituye la conciencia del tiempo. ¿Qué son esas intenciones básicas?

Esas intenciones básicas son aquellas que figuran en lo que Husserl llama la impresión originaria del ahora: a saber, su referencia a lo que ha sido y a lo que aún está por ser. Sin embargo, todavía permanece la dificultad: ¿cómo podría ese horizonte retencional y protensional de la impresión originaria del ahora servir para constituir el acto en el sentido de conferirle su intención, que es lo que hace al acto un acto? Lo que la consideración de Husserl logra manifestar es cómo un continuum de acto es posible. Ello 
muestra cómo el flujo de la vida consciente, a la que esos actos pertenecen en un sentido, es hecho posible. Pero más allá de esto, no explica el carácter activo de los actos. Ahora bien, yo no deseo afirmar que ningunos actos son ulteriormente reducibles, sino que deseo mantener que la especie de intención, que caracteriza los actos, no es reductible a la especie de intención, que caracteriza la impresión originaria del ahora en el análisis de Husserl sobre la conciencia del tiempo. Por supuesto, una intención compleja de la primera especie, es analizable en intenciones más simples de la misma especie, es decir, en otras intenciones-acto, sobre las cuales está basado, pero yo no veo cómo una intención-acto es reducible a intenciones, que no sean intenciones-acto.

De este modo, la teoría de la constitución de los actos en la conciencia del tiempo original falla a causa del hecho de que los actos no son temporales en el mismo sentido en que lo son los objetos temporales durables, y también porque la especie de intención que caracteriza los actos o más bien los hace lo que son, no es reducible a la especie de intención que caracteriza a los datos básicos de la conciencia del tiempo.

Por supuesto, los actos están en el tiempo en el sentido de que pueden ser situados temporalmente, y también en el sentido de que ciertos predicados temporales son valederos de ellos. Por ejemplo, podemos decir que un cierto acto fue temporalmente antes de algún otro acto, o después de él. Pero muy curiosamente algunos otros predicados temporales no se les pueden atribuir con pleno sentido: no podemos, por ejemplo, decir que mi acto de percepción de esta casa frente a mí atraviesa por varias fases temporales. Esta importante diferencia no debería pasar inadvertida. Este último punto, según parece, es el que sirve de fundamento a la insistencia de Gilbert Ryle de que ciertos verbos mentales no expresan episodios, sino acabamientos.

Hay otro camino para abordar la pregunta sobre una posible constitución de los actos. Procurando adherirse al paralelismo entre el noema trascendente y los actos, puede uno sostener que así como el noema trascendente es constituido por los actos actuales y posibles, a través de los cuales llega a ser dado o se tiende a él, así puede decirse que un acto es constituido por los modos de su ser-dado. ¿Cómo, pues, es dado un acto? Un acto es una Erlebnis, es inmediatamente dado, de hecho es auto-dado. Es, según sú verdadera existencia, dado. Por tanto, sería autoconstitutivo. Sin embargo, puede todavía argüirse que un acto puede ser dado como objeto para una reflexión de orden superior, y la pregunta sobre su constitución aparece sólo en cuanto que puede ser dado como objeto. Bien, luego la constitución de un acto sería buscada en la reflexión que la hace su objeto; pero entonces sólo estamos reduciéndola a otra intención-acto, pues la reflexión es otro acto, si bien de un orden superior. No logramos ir más allá de las intenciones de 
acto, no tenemos éxito en alcanzar alguna otra especie de intención como constitutora del acto.

Fink, en Vergegenwärtigung und Bild (1930) ve la distinción entre intencionalidades-acto e intencionalidades que no son actos. Al último tipo pertenecen, por ejemplo, retenciones e intencionalidades-horizonte. Un punto de distinción entre los dos grupos, de acuerdo con Fink, es que las intencionalidades que no son actos, son unselbständig, no pueden existir solamente por ellas mismas, sino que han de adherirse siempre a algo distinto, mientras que las intencionalidades-acto son en un sentido experiencias selbständig. Además la primera clase de intencionalidades no posee en sentido estricto un objeto intencional, si bien "constituyen la 'condición de posibilidad' de toda objetividad: el horizonte temporal, fuera del cual algo semejante a un objeto, como identidad que se mantiene a sí misma a través del flujo de las fases temporales, pudo absolutamente emerger".10

Es significativo desde nuestro punto de vista que Fink hable del horizonte temporal y sus intencionalidades, como constitutivas de las condiciones de posibilidad de una intención-acto como tendiente a un objeto. La concepción de un objeto es, por supuesto, la de una identidad que persiste a través del tiempo, y sólo en cuanto que hay un horizonte temporal constituido por sus intencionalidades básicas como retención y protensión, es posible tender a un objeto como éste. En esa forma las intenciones básicas de nuestra conciencia del tiempo sustentan las condiciones de posibilidad de los objetos y así, también, de los actos que tienden a tales objetos en cuanto que tienden a ellos, es decir, en cuanto que son intenciones-acto. Pero decir que por eso hemos alcanzado las condiciones de posibilidad de los actos no es lo mismo que decir que hemos expuesto la constitución de los actos. Nosotros estamos más cerca de Kant, y sostenemos que la temporalidad, como constituida por las intenciones básicas que no son actos, hace posible para nosotros tender a objetos, pero ellas solas no constituyen ora los objetos, ora los actos.

Las observaciones anteriores no tienen el sentido de apoyar la tesis de que las únicas especies de intenciones de que podemos hablar legítimamente, son las intenciones-acto. Lo que ellas se proponen iluminar, es el hecho de que las intenciones-acto no son, en un sentido muy importante, de ningún modo ulteriormente reducibles.

\section{III}

Con respecto al llamado dato hilético, las lecciones sobre la conciencia del tiempo empiezan efectivamente con el punto de vista de las Ideen I: se nos dice, por ejemplo, que el rojo sensible es un dato fenomenológico, que, cuan-

10 Fink, Studien zur Phänomenologie, The Hague, 1966, p. 25. 
do está animado por una cierta función de aprehensión, presenta una cualidad objetiva. ${ }^{11}$ Pero las lecciones proceden inmediatamente a sugerir una cierta teoría de la constitución del dato mismo. Sobre este punto las lecciones muestran una contradicción interna, una tergiversación de puntos de vista, que perturba un poco, y ahora debemos a Boehme haber aclarado que la edición de Heidegger de las lecciones sobre la conciencia del tiempo (de las cuales el trabajo edicional había sido realizado hacía mucho por Edith Stein) incluye los escritos de Husserl sobre el tema, pertenecientes a diversos periodos y que representan por ello estratos muy diversos de concepciones que no son todas mutuamente compatibles.

De hecho, aparte de la repetición de concepciones tempranas en cuanto a la naturaleza del dato según se ejemplifica en el pasaje citado, tenemos otras dos concepciones muy diferentes:

En primer lugar, tenemos la sugestión ${ }^{12}$ de que el dato sentido, si bien anterior a su ser animado por el acto de aprehensión, puede ya haber sido constituido. Esta concepción es elaborada ulteriormente en el Apéndice V, que pertenece a una fecha posterior (lo cual sugiere que la nota al pie citada arriba, ¡no puede ser de la misma fecha que el texto a la que fue añadidal), donde se nos dice que el dato tiene ya que haber sido constituido anteriormente al acto que ha de animarlo, y la razón que se aduce en favor de esta concepción parece ser más o menos como sigue: el dato total al que se aplica el acto o al que se supone animar el acto, es una totalidad que tiene duración y que por ello consiste en varias fases. Estas varias fases requieren ser mantenidas juntas y formadas en un dato total, y ello se hace posible en virtud del horizonte intencional de cada sensación originaria del ahora. Y obviamente, esa intencionalidad que se adhiere a toda sensación originaria del ahora, no es una intencionalidad-acto. Lo que Husserl descubre por ese medio es la necesidad de cierta clase de síntesis, que hace posible que el dato total esté presente como una totalidad, como un dato, para que el acto de aprehensión trabaje sobre él. Esto no habría sido posible, si cada dato momentáneo estuviera completamente desconectado de otro; los ahora discretos nunca formarían un todo, sólo habría momentos perecederos. El dato total, como la totalidad de las fases transitorias, se hace posible simplemente porque cada momento no es un átomo discreto, sino una estructura intencional que tiene su propio horizonte retencional y protensional.

La intencionalidad del dato hilético no significa que el dato es de algo, pues esa referencia al objeto trascendente primero se hace posible a través de la animación por el acto. El acto, el acto objetivante en particular, es la fuente de la referencia al objeto trascendente. El dato hilético qua dato es intencional en un sentido muy diferente, a saber, en el sentido de que

$11 \mathrm{ZBW}, 371$.

$12 \mathrm{ZBW}, 371$ nota. 
apunta más allá de él mismo al pasado y al presente: su intencionalidad es simplemente su temporalidad. A pesar de los adelantos efectuados en las lecciones sobre la conciencia del tiempo, el dato hilético sigue siendo no-intencional en el sentido en el que se dijo que lo era en las Ideen I, si bien ahora se descubre una nueva clase de intencionalidad en su estructura interna. Seguramente no poseen ellos intencionalidad-acto, más bien la reciben del acto.

De este modo, parecería que, aun cuando son introducidas algunas nuevas concepciones de la intencionalidad, no se reclama ningún cambio fundamental en el esquema de las Ideen I. Lo que se reclama son alteraciones menores y cambios de perspectiva, en suma, una consideración de todo el asunto a la luz de la estructura de la temporalidad que lo penetra todo.

Sokolowski, como se dijo antes, acusa a Husserl de ofrecer un análisis formal del tiempo que (i) fracasa en dar razón de la cualidad de los actos como unidades constituidas, y (ii) fracasa en dar razón del aspecto material, es decir, de contenido de los datos hiléticos. ${ }^{13} \mathrm{Y}$ todavía Sokolowski rechaza el dualismo de Husserl entre el contenido no-temporal y la forma temporal de los objetos sensibles, como también su dualismo entre datos hiléticos y actos que los animan. De hecho, parece favorecer la tendencia monista de los escritos posteriores de Husserl. ${ }^{14} \mathrm{~A}$ mí me parece, sin embargo, que alguna especie de dualismo es necesaria para dar razón de la facticidad de las unidades constituidas, sin atender a si son objetos trascendentes o datos hiléticos: no todo su contenido puede derivarse de su temporalidad. Aun cuando no estemos satisfechos con un dualismo absoluto entre forma y materia, podría muy bien haber una especie de dualismo relativo, en el cual lo que funcionó como acto dador de significado en un nivel inferior, puede justamente ser la materia para una unificación ulterior en un nivel más alto. Una intención que va sobre todas puede usar las intenciones más primitivas como su materia, de modo que en cualquier nivel la distinción entre forma $y$ materia tiende a emerger. El esquema forma-materia, en consecuencia, no nccesita ser abandonado. Puede ser realmente el esquema más universal ilustrado en nuestra experiencia en todos los niveles. Decir que ninguna constitución tiene la forma $A$ uffassungs-Inhalt, sólo significaría entonces que no toda forma es conferida por la intención-acto, que hay especies totalmente diferentes que dan significado, síntesis o unidad..$^{15}$

El problema crucial para todo fenomenólogo es de hecho cómo retener este dualismo sin (a) sucumbir a un fácil empirismo sensualista con respecto a la hyle (que es lo que encontramos en Kant, por lo menos en partes de la Estética trascendental), (b) sin escapar subrepticiamente de la epoché (y por

13 Sokolowski, loc. cit., pp. 92-93, 100, 114-115.

14 Ibid., pp. 98, 105.

15 Compárense los comentarios de Iso Kern a la caracterización que hace Fink de la temprana distinción de Husserl entre hyle y acto intencional como si fuese meramente provisional, en su Husserl und Kant (Phenomenologica, 16) The Hague, 1964, p. 274 . 
ese medio apelar a explicaciones causales), y (c) finalmente sin identificar todas las intenciones con intenciones-acto, todas las sintesis con síntesis activas, toda constitución con un moldeamiento intelectual. Ésta es sin duda una tarea formidable, pero no la podemos rehuir, pues la alternativa nos conduciría a la absurda interpretación de la constitución como creación.

Las lecciones sobre la conciencia del tiempo afirman -dejando a un lado las teorías de la constitución de los actos y datos hiléticos- la teoría de la autoconstitución del flujo absoluto de la conciencia. Ahora podemos tratar de entender esta muy importante tesis. El flujo absoluto de la conciencia, en el que suponemos ser constituidas todas las unidades inmanentes y trascendentes, no está él mismo en el tiempo, no es una unidad constituida, no es un objeto durable en el tiempo, sino que es la fuente original de la conciencia del tiempo $y$, así, de todo tiempo, objetivo e interno. Husserl nos da un número de grados sucesivos - que no son enteramente los mismos en cuanto reducciones sucesivas - por los cuales podemos llegar a exhibir ese flujo de la conciencia en su pureza original. Supongamos que yo escucho una melodía, un sonido continuo. En ese caso estoy viviendo en la audición del sonido por decirlo así. Pero, en lugar de vivir en ese acto, puedo atender al sonido en cuanto que es escuchado, en cuanto me aparece, es decir, como un dato fenomenológico. Después puedo atender a las continuidades del matizado de las diferentes fases de ese fenómeno de sonido. Finalmente, puedo atender no a las continuidades en el matizado de las fases del fenómeno de sonido (que es una unidad inmanente constituida), sino a la continuidad en el matizado de mis aprehensiones de esas fases, por lo cual, estaría atendiendo al flujo de la conciencia misma. Ahora bien, esto puede sugerir que el flujo puede llegar a ser un objeto de reflexión o atención, o aun de retención. Mas, primeramente, esas mismas reflexiones, atenciones o retenciones pertenecen al flujo mismo, de manera que lo que tenemos es el notable fenó. meno del flujo que retorna sobre sí mismo. En segundo lugar, el flujo es dado como flujo aún anterior a su ser reflexivamente atendido. "Aunque la reflexión no se lleva a cabo ad infinitum y aunque, en general, la reflexión no es necesaria, sin embargo, ha de ser dado lo que hace posible esa reflexión y, en principio (o así lo parece, por lo menos), posible ad infinitum." 16

Cada Erlebnis separada se da también reflexivamente antes de la objetivación, pero se da sólo en cuanto que forma un constituyente de ese flujo de la conciencia. El flujo es el que se da primariamente así, la Erlebnis ais.

16 "Wenn nun auch nicht in infinitum Reflexion geübt wird und überhaupt keine Reflexion nötig ist, so muss doch dasjenige gegeben sein, was diese Reflexion möglich macht und, wie es scheint, prinzipiell wenigstens in infinitum möglich macht." ZBW, 468 . 
lada no se da como aislada, sino como perteneciente al flujo. En consecuencia, únicamente el flujo es auto-dado. Sólo él elude por completo la objetivación, pues en ese preciso intento de objetivarlo, retorna a sí mismo como el flujo subyacente. Parece no haber medios de situarse fuera del flujo y de hacerlo objeto, pues ese preciso acto de objetivar pertenecería él mismo al flujo.

Siendo, de esa manera, auto-dado en el sentido estricto, el flujo es también auto-constitutivo. Pues constituye su propia unidad en virtud de intenciones pertenecientes a él mismo. En virtud de lo que Husserl llama "intencionalidad longitudinal" (Längsintentionalität), el flujo está "en continua unidad de coincidencia consigo mismo". ${ }^{17}$ Cuando cada sensación primaria se cambia en una retención, una nueva sensación primaria viene a unirse a esa retención y asimismo en el tercer momento una nueva sensación primaria viene a unirse a la retención de la retención de la primera y retención de la segunda. Y las retenciones de retenciones, cualquiera que sea el grado de su complejidad, contienen todavía una referencia intencional a la sensación primaria, que es, por decirlo así, su punto originador. En virtud de tales propiedades inmanentes al flujo es por lo que el flujo aparece a si mismo como flujo, sin requerir otro flujo en qué aparecer.

El flujo original de la conciencia es descrito de varias maneras. Se lo llama conciencia original del tiempo, también se lo llama el flujo absoluto de la conciencia. Lo que necesitamos para tener claridad al respecto es el sentido preciso de su temporalidad y de su absolutez. Es obvio, por supuesto, que él no está en el tiempo, ni en el tiempo trascendente ni en el tiempo inmanente. En otras palabras, los predicados temporales, como comienzo y fin, antes y después, no pueden aplicarse con plenitud de significado al flujo como flujo. Además, éste no dura, pues todos los objetos durables son unidades constituidas. En ese sentido tampoco persiste. No puede asimismo ser concebido a la manera de un cambio objetivo, consistente en series de fases durables, cada una de las cuales puede ser identificada como objeto sea trascendente sea inmanente. Nada persevera en ese flujo, salvo y excepto la estructura formal del flujo, es decir, el nexo de retención-protensión del ahora, no cualquier nexo particular semejante, sino sólo su simple forma. ${ }^{18}$ Por esto Husserl habla correctamente de él como conciencia intemporal, ${ }^{19}$ y rehúsa caracterizarlo como proceso. ${ }^{20}$

¿En qué sentido es él absoluto? Reuniendo las observaciones de las

17 ZBW, 435 .

$18 \mathrm{ZBW}, 466-467$.

19 "Die subjektive Zeit konstituiert sich im absoluten zeitlosen Bewusstsein, das nicht Objekt ist." ZBW, ${ }_{4} 6_{4}$.

20 Husserl pregunta: "Kann man hier im eigentlichen Sinn von einer Veränderung sprechen, wo doch eine Unveränderung, eine unverändert ausgefüllte Dauer undenkbar ist?" $\mathrm{ZBW},{ }_{4} 66$. 
Ideen I con las de estas lecciones sobre la conciencia del tiempo, encontramos las siguientes caracterizaciones del flujo de la conciencia, las cuales pueden ayudarnos a entender precisamente en qué sentido puede decirse que es absoluto.

El flujo de la conciencia es indubitablemente dado, y no admite ilusión, ser-de-otra-manera, o duda. ${ }^{21}$ Es dado sin variaciones de apariencia o perspectiva. ${ }^{22}$ Es una esfera de ser auto-contenida, en la cual no puede entrar nada de fuera y de la cual nada puede salir, que es independiente y no posee un mero ser intencional, como es el caso de todos los objetos trascendentes (e inmanentes). ${ }^{23}$ La epoché no afecta su naturaleza esencial, aun cuando disocia al flujo de sus adherencias naturalistas. Además, él es la fuente de significados, el reino de los "orígenes absolutos"; constituye todas las objetividades, pero él mismo es auto-constitutivo. ${ }^{24} \mathrm{La}$ denominación de "absoluto" puede tomarse como sustitutiva de todas esas características del flujo de la conciencia.

Sin embargo, debería recordarse que aun este flujo de la conciencia no es adecuadamente dado, pues todavía tenemos que "nadar detrás de" las Erlebnisse en él, y apoderarnos de ellas mediante cierta clase de memoria retrospectiva. Esto, al menos, parece ser lo que Husserl dice en las Ideen I. ${ }^{25}$ En las lecciones sobre la conciencia del tiempo, uno de los puntos ganados en este respecto es que la retención primaria no es un recuerdo retrospectivo, sino una aprehensión directa del pasado como pasado, de hecho es una percepción, en la cual lo que-acaba-de-ser es visto directamente. ${ }^{26}$ Esto reduce la dificultad creada por la naturaleza del flujo, que nos exige "nadar detrás" de ël. No obstante, jamás llegamos a tener una aprehensión total del flujo sub specie aeternitatis, pero estamos dentro de él aun cuando lo sepamos, pues nuestro saber es también una parte de ese flujo. Esto excluye un modo de ser dado, completamente adecuado, del flujo en cuanto flujo, si bien en cuanto se da, se da inmediata y directamente, y no a través de perspectivas o apariencias.

Podemos distinguir, siguiendo a Husserl, entre (i) el tiempo objetivo al que pertenecen los objetos trascendentes, (ii) el tiempo inmanente - también objetivo, que es constituido- al cual pertenecen las unidades inmanentes constituidas, como actos y datos hiléticos, y (iii) el flujo absoluto de la conciencia, que es la fuente de toda temporalidad y que no podemos decir que él mismo está en el tiempo. En adición a esta lista, necesitamos reconocer

21 Edmund Husserl, Ideas. (Las referencias son a la edición en rústica de la traducción inglesa de Boyce Gibson), 191.

22 Ibid., 198.

23 Ibid., 139.

24 Ideas, 153-154; ZBW, 434.

25 Ideas, 127.

$26 \mathrm{ZBW}, 392-395$. 
un nivel de temporalidad que debe colocarse entre (ii) y (iii), y que ha de explicar cómo (iii) se constituye él mismo en (ii). Éste es el nivel de lo que Husserl llama tiempo "pre-fenoménico" o "pre-inmanente", en el cual las fases del flujo están dispuestas de una manera más bien "quasi-temporal" (quasi zeitlich).27 Así, mientras el flujo absoluto de la conciencia no es en modo alguno temporal y de hecho es intemporal, esto en virtud de su propia estructura intencional, se da él a sí mismo como ordenamiento quasi-temporal de sus fases, y en este ordenamiento quasi-temporal, en este tiempo pre-inmanente, es donde las objetividades inmanentes y el tiempo inmanente son constituidos. Sin embargo, los dos, a saber, el flujo absoluto no temporal y el ordenamiento quasi-temporal, no son idénticos, ni son diferentes. El último es el modo como el primero aparece a sí mismo. ${ }^{28}$

Estos comentarios sobre algunos aspectos de las lecciones de Husserl sobre la conciencia del tiempo se proponen llamar la atención sobre ciertas limitaciones de una teoría fenomenológica de la constitución. Negativamente, estos comentarios se proponen dar claridad al hecho de que constitución no es creación, de que fenomenológicamente es inevitable alguna clase de dualismo. Positivamente, apuntan a la percepción clara de que la constitución podría entenderse de modo que no anule las distinciones fundamentales entre trascendencia e inmanencia, entre actos y no-actos, entre intencionalidadesacto e intencionalidades que no son actos, entre la corriente temporal de las Erlebnisse y la conciencia absoluta no-temporal, que puede decirse que es un flujo sólo en un sentido altamente figurativo y analógico del término. Las distinciones esenciales y las discontinuidades resultantes no deberian pasar inadvertidas, y una teoría de la constitución solamente puede correr el riesgo de dejar de ser fenomenológica, si no tiene éxito en tener cuidado de ellos.

J. N. Mohanty

(Trad. de Bernabé Navarro)

UNIVERSIDAd DE BURDWAN, INDIA

27 ZBW, 436.

28 Scheler distinguía entre la experiencia vivida y el vivirla, y sostenía que aunque la experiencia vivida es temporal, el vivirla es no-temporal. Fundamentalmente, nuestra interpretación de Husserl sobre este punto conviene con Scheler, excepto que a nosotros no nos agrada separar los dos niveles tan radicalmente como lo hace Scheler. Para nosotros, la experiencia vivida es lo que el vivirla aparece a sí mismo de acuerdo con su naturaleza intrínsecamente intencional. Éste es el punto de vista que atribuiríamos a Husserl en ese estadio. Compárese y contrástese con Mikel Dufrenne, The Notion of the Apriori, E. Tr. by Edward S. Casey, Northwestern University Press, 1966. 